\title{
On the frequency measurement in Wide Area Measurement and Control Systems
}

\author{
Mihai Sănduleac ${ }^{1}$, Ana-Maria Dumitrescu ${ }^{1}$, Lucian Toma $^{1}$, Carmen Stănescu ${ }^{2}$, Mihaela Albu ${ }^{1}$ \\ ${ }^{1}$ Politehnica University of Bucharest \\ ${ }^{2}$ Transelectrica Company \\ m.sanduleac.ro@ieee.org,anamaria.dumitrescu@upb.ro,lucian.toma@upb.ro,albu@ieee.org
}

\begin{abstract}
Emerging power systems are requiring changes on all layers: planning, operation and markets. Smart grids operation, including control of the energy flow in active distribution grids adds more challenges to the measurement layer. Presently there is a gap between the models used for the current and voltage waveforms in the corresponding measurement algorithms implemented by devices deployed in power systems and the functionalities (like choice of available reporting rates) of the new generation of synchronized measurement units (SMU). Overlooking these issues can determine a significant depreciation of the information mediated by the control systems that are relying on real-time measurements delivered by equipment. Moreover, several applications require an estimation of the measurement quality thus enabling an effective control: state estimation, power quality improvement, voltage control, frequency control, network parameter' estimation etc. Wide Area Measurement and Control Systems (WAMCS) have to accommodate multiple measurement approaches: on one side, the existing time-aggregation based measurements offered by currently deployed Intelligent Electronic Devices in SCADA framework, including smart meters and other emerging units; while on the other side, the high-resolution waveform-based monitoring devices like Phasor Measurement Units (PMUs) with fault-recorder functionality. In this paper the case of frequency measurement is analysed, addressing the measurement paradigm on a long term vision of future energy systems.
\end{abstract}

Keywords: PMU, smart meter, frequency measurement, SCADA

\section{INTRODUCTION}

The ever-increased share of variable renewable energy sources in existing electricity systems (i.e., inheriting a classical network infrastructure) challenges the existing models and control paradigm. Moreover, technological advancements in Information and Communications Technology (communication, data compression, encryption, GPS availability, cloud-based operation, Internet of Things concept etc.) make possible large scale deployment of new measurement equipment and real-time processing of synchronized measurements.

Presently, there are several issues that require a fundamental new approach:

- closer to quasi-zero inertia power system due to power converters mediated energy transfer requires new control algorithms able to cope with smaller time constants (order of milliseconds even in steady state conditions). This can be achieved with a measurement layer with higher dynamic performances and algorithms able to discriminate normal operation from faults by adapting the measurement set-up accordingly, for example by using higher reporting rates. Merging measurement data made available with different reporting rates and/or embedding standard aggregation algorithms is associated with an evaluation of the quality of measurements; this needs to consider definitional uncertainty, strongly dependent on the physical model of the energy transfer in the considered grid section. Moreover, it becomes useful to quantify the measurement data adequacy to the phenomena monitored by performing an assessment of the impact of heterogeneous reporting rates measurements [1].

- societal demand for anywhere - anytime energy availability and quest for sustainability and resilience has accelerated deployment of microgrids, which can operate either stand alone or in grid-connected mode. The basic principle is balancing energy generation and consumption at the lowest possible level. This approach allows a hierarchical architecture where each level solves at its best the internal unbalance and gives a lowest need for power balancing at higher levels. Moreover, d.c. power distribution systems are taking ground, mostly as part of larger hybrid (AC/DC) systems. The energy exchange between [hybrid] microgrids and the electrical grid is bidirectional, needs a high resolution of the time axis for the electric quantities and new regulatory framework.

- larger deployment of synchronized measurements faces the barrier of cost and information retrieval provided that suitable models for the energy transfer are developed. To support the demanding requirements of the future energy systems latencies at levels below 10ms (the coming generation of $5 \mathrm{G}$ networks) will be essential. Meanwhile, high reporting rate dynamic measurements from ubiquitous new generation of smart metering and communication infrastructures can be intelligently exploited in hybrid state estimators. Although Distribution System State Estimation (DSSE) is already an established technology [2], its deployment is seldom used due to computational complexity, especially in active distribution networks [3].

\section{SYNCHRONISED MEASUREMENT SYSTEMS}

We consider in our assessment three main types of measurement devices: the phasor measurement unit (PMU), the SCADA measuring device and the energy meter.

All these measurements devices have a certain synchronisation level, thus are appropriate for being used in a hybrid measurement system for feeding the real-time smart grid applications. PMUs and the recently deployed in distribution networks microPMUs have synchronisation accuracy in the order of microseconds, due to GPS-based 
timing control. This precise synchronisation algorithm is a requirement for voltage estimation in phasor form, as well as for the frequency (and its derivative). Moreover, it can be ensured high reporting rates up to 100 frames/s [4].

Presently traditional SCADA equipment have also synchronisation procedures which allow them to cope with milliseconds timeframe for addressing the event -driven timestamps; however the measurements further used in control algorithms (not protection) such as: active and reactive power, currents and voltages (rms values), or frequency, are not subject of a standardized algorithm, in what concerns both the reporting rate and the aggregation procedure.

The so-called internal reporting rate is usually not synchronized with the internal clock (used for other SCADA enabled services). Reporting rates of 1 or 2 measurements per second for SCADA can be reached by some Remote Terminal Units (RTU) / Bay Control Units (BCU) or Intelligent Electronic Devices (IED), however reporting rates on the whole chain from e.g. a substation and a control center can be in the range of several seconds ( 2 to 5 seconds). At the control center level we can rely on a generic $3 \mathrm{~s}$ reporting time intervals between data frames arriving from different network measurement nodes.

Energy meters can be externally synchronized at the level of 1 second, usually by means of meter specific protocols (e.g. DLMS). Although internally the computation of energy values can use several real-time measurements per second, the accessible reporting time for external readout of such real-time (instrumentation) values is usually in the range of 1 to 5 seconds. The full chain readout of such data by a control center with metering front-end may have similar time uncertainty for the synchronisation of received measurements.

Recently, by using a Unbundled Smart Metering concept, a synchro-SCADA approach has been tested for having a synchronisation down to one second between any such meterbased equipment [5-7].

In order to analyse the quality of real-time data, the resolution and accuracy of measurements have to be considered. PMUs and microPMUs have specified accuracies and high resolution for the real-time data such as frequency, rocof and phase. Particularly the frequency measurement has usually associated the accuracy of $1 \mathrm{mHz}$, which makes it appropriate for monitoring and controlling the volatile active distribution grids.

In Table I the characteristics of different commonly used measurement devices, with focus on the frequency measurement, are synthetically presented. The best characteristics are obtained by PMUs/microPMUs and the next candidate is the Unbundled Smart Meter [6]. We focus our analysis on these two types of equipment.

\section{TIME- AND SPACE AGGREGATION OF SYNCHRONIZED MEASUREMENTS}

In order to compare frequency measurements we consider three devices connected to the same grid LV grid node (i.e. no additional instrument transformer in the measurement chain):

- One PMU [8]

- One microPMU [4]
- One Unbundled Smart Meter using a ZMD405 meter as Smart Metrology Meter [6, 9].

The comparison has been made for a measurement window including dynamic conditions, when the grid frequency had a maximum deviation in the range of a $100 \mathrm{mHz}$, corresponding to a sudden power unbalance in the Romanian network (part of the ENTSO-E operated power system) larger than $500 \mathrm{MW}$. Fig. 1- 4 show the evolution of each measurement for a 10 minutes window including the event.

Fig. 1 gives a general picture over 10 minutes, with the synchronized data from the frequency measurements reported by all three devices: microPMU, PMU and Unbundled Smart Meter (USM). It can be seen that both microPMU and PMU detect the disturbance and associate it with higher frequency deviations than the meter does.

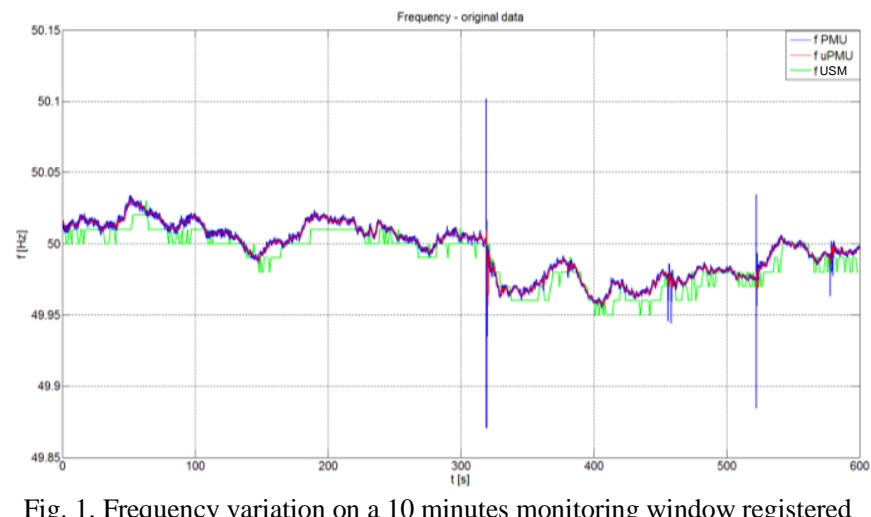

Fig. 1. Frequency variation on a 10 minutes monitoring window registered with PMU, microPMU and USM

This is the result of the dynamic capabilities of the three devices, as reflected by the maximum available (and selected) reporting rate: PMU is set on 50 frames/s (successive data frames every 20ms), microPMU is set on 100 frames/s (successive data frames every 10ms), and USM is set on 1 frame/s (successive data frames every $1 \mathrm{~s}$ ).

Once can see that for steady state conditions, the high resolution for frequency measurement ensured by the USM allows good correlation with the more accurate devices (PMUlike). The average deviation of USM measurements, compared with those from the more sophisticated PMUs, is only half of its resolution, meaning $5 \mathrm{mHz}$, which is a good achievement for such a measurement equipment.

Fig. 2 gives a detail of the frequency variation during disturbance. It can be seen that the microPMU captures well the transitory period of the disturbance, when it shows oscillations associated with the dynamic behaviour of the power system. These oscillations are not captured well by the USM, due to its embedded aggregation algorithm.

However, this feature makes USM useful in processes with time-reaction of seconds or tenths of seconds, such as the frequency containment reserve (FCR), where technical minimum requirements, according to Article 154 and its Annex 5 of [10], ask for full FCR activation in 30 seconds and for a minimal accuracy of frequency measurement of $10 \mathrm{mHz}$, which are well covered by the $1 \mathrm{~s}$ reporting period for frequency and by the $5 \mathrm{mHz}$ deviation as has been shown above. 
TABLE I.

COMPARATIVE CHARACTERISTICS OF DIFFERENT MEASUREMENT EQUIPMENT

\begin{tabular}{|c|c|c|c|c|c|}
\hline Description & PMU & Micro PMU & RTU/ BCU/ IED & Classic Energy meter & Unbundled Smart Meter \\
\hline Synchronisation requirements & $<1 \mu \mathrm{s}$ & $<1 \mu \mathrm{s}$ & $1-2 \mathrm{~s}$ & $1-5 \mathrm{~s}$ & $\leq 1 \mathrm{~s}$ \\
\hline Reporting rate (typical) [frames/s] & 50 & 100 & 1 & $1-0.2$ & $>1$ \\
\hline Freq. resolution in steady state conditions [mHz] & $<0.01$ & $<0.01$ & $10 \ldots 100$ & $10 . .3100$ & 10 \\
\hline Accuracy & Spec. & Spec. & Not spec. & Not spec. & $\approx 0.2 \%$ \\
\hline Measurement capabilities & Dynamic state & Dynamic state & Steady state & Steady state & Steady state \\
\hline
\end{tabular}

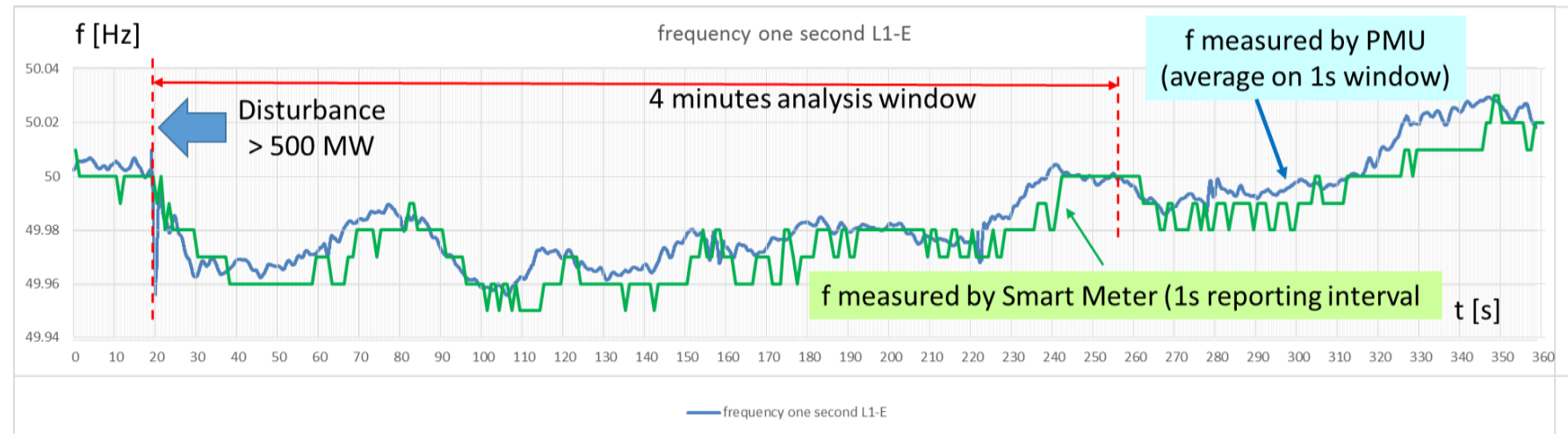

Fig. 2. Frequency during the power system disturbance: microPMU measurements (100 frames /s rate) and USM measurements.

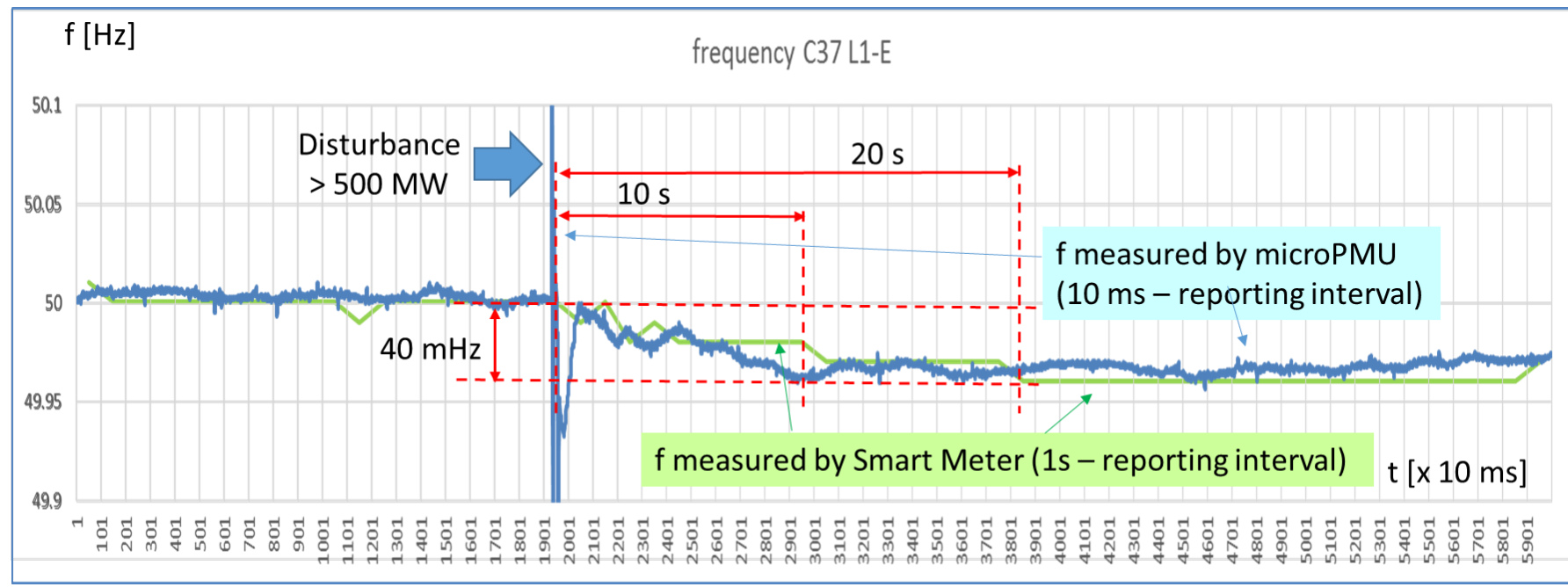

Fig. 3. Frequency during power system disturbance: microPMU data aggregated (asynchronous 100 points average) and USM data (original measurements)

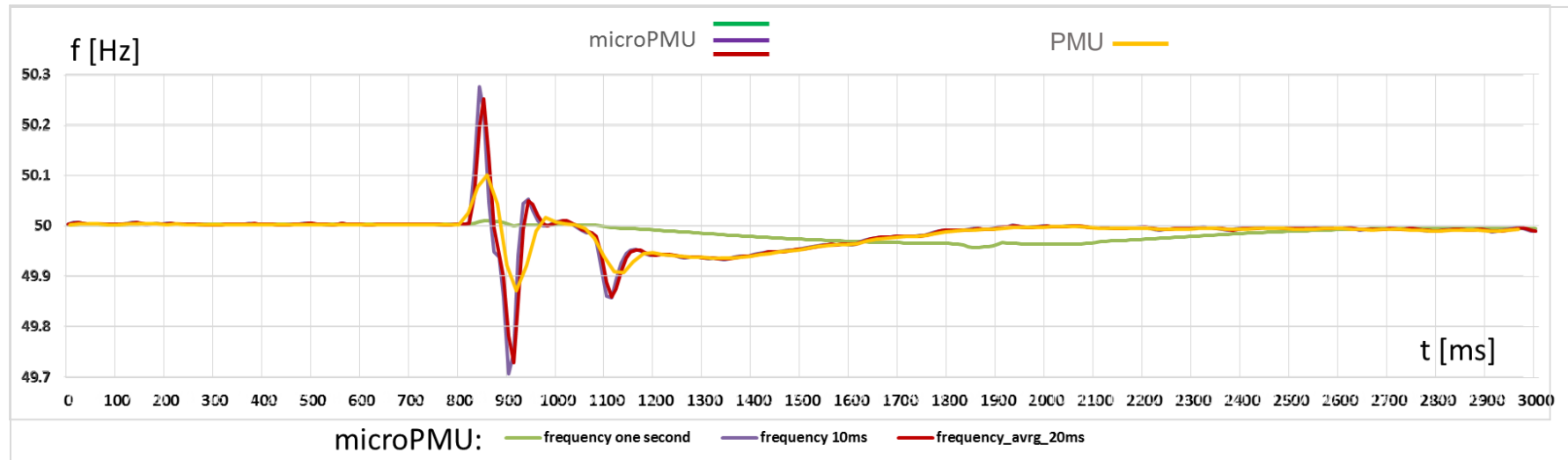

Fig. 4. Frequency during power system disturbance: microPMU (asynchronous 100 points average with reporting interval of $1 \mathrm{~s}$; original data with 100 frames/s; asynchronous 2 points average with reporting interval of 20ms) [4]; PMU data (50 frames/s) [8] 
Fig. 3 captures the frequency evolution during a 6 minutes monitoring for one second averaged microPMU and for USM frequency evolution. It shows comparatively the $1 \mathrm{~s}$ averaged data from microPMU and the USM reported values (1 frame/s).

It can be seen again that the USM measurements are well in line with the PMU measurements, capturing the pattern of the frequency evolution on a second-based sampling. The figure suggests again that the meter can be a reliable source of local frequency information for different applications where the time constant of the control loop (including decision making) is in the order of seconds, such as frequency containment reserve and other balancing mechanisms, especially in microgrids.

The biggest advantage of using the meter (instead of traditional synchronized measurement devices, PMU-like), beyond price affordability, is the fact that this is anyhow present at prosumer side for billing purposes and can be used also for such additional grid operation tasks.

Fig. 4 presents more detailed frequency information derived from microPMU and PMU data reported during the disturbance, on a time window of $3 \mathrm{~s}$. The microPMU has twice the reporting rate of the PMU and data are further mediated as to emulate lower rates: 1 frame/s. We also emulate the reporting rate of PMU (50 frames/s).

As the market trend is to have higher maximum reporting rates (100 frames/s or even larger), the different types of application for all the acquired data implies the need for a dedicated, application dependent processing of information.

Using averaging algorithms for data aggregation in order to achieve data compatibility between data flows originally available with different reporting rates (and following different frequency estimation algorithms) is actually introducing additional filtering, which is associated with computational delay and information distortion (green line minimum value shows (Fig. 3) a delay of around $800 \mathrm{~ms}$ from the moment of disturbance), which may be badly accommodated in some SCADA applications.

It can be seen that the microPMU has a better dynamic behaviour during the initial transient period, with clear advantages in quasi-steady state control applications like dynamic state estimators.

The USM synchronization of 1 second was sufficiently good in order to pursue the frequency series measured with the PMU, thus showing that for grid services such as contributing at primary reserve with small resources the USM may be a good candidate of the necessary measurements and actions.

\section{CONCLUSION}

The paper analyses the frequency measurement performed with three types of equipment: PMU, microPMU and Unbundled Smart Meter. The analysis of a power network disturbance shows that each measurement paradigm has its own advantages: PMU and microPMU capture in detail fast frequency disturbances, within tenths and hundreds of milliseconds, which brings a good understanding of the disturbance effect in the network, while the reporting rate of one second of the smart meter gives good-enough frequency information for quasi-steady state applications (1s-control loops), while additionally being compatible with existing SCADA measurements, the reaction time and accuracy needed for the cost-effective implementation of frequency containment reserve at various levels of the network is to the considered before choosing the measurement set-up.

\section{ACKNOWLEDGEMENT}

The authors gratefully acknowledge the support of European Union's Horizon 2020 research and innovation programme under the grant agreement No 727481 (RESERVE Project).

\section{REFERENCES}

[1] Mihaela Albu, Ana-Maria Dumitrescu, ,Applicability of synchronised measurements in modern distribution grids. A discussion", Proc. of IEEE Applied Measurements for Power Systems AMPS2014, Aachen, 24-26, Sept. 2014

[2] C. Muscas, P. A. Pegoraro, S. Sulis, M. Pau, F. Ponci, A. Monti, "Fast multi-area approach for distribution system state estimation", IEEE I2MTC - 2016 International Instrumentation and Measurement technology Conference, May 23-26, 2016, Taipei, Taiwan.

[3] Georgia Pieri, Markos Asprou, Elias Kyriakides, "Load pseudomeasurements in distribution system state estimation”, Proc. of 2015 IEEE Eindhoven PowerTech, June 2015, Eindhoven, The Nederlands.

[4] PowerStandard Labs, $\mu$ PMU Installation and User's Manual, available to download at:

http://www.powersensorsltd.com/PQube3.php\#technical

[5] Mihai Sănduleac, Lucas Pons, Giampaolo Fiorentino, Rares Pop, Mihaela Albu - The unbundled Smart Meter concept in a synchroSCADA framework, IEEE I2MTC - 2016 International Instrumentation and Measurement technology Conference, May 23-26, 2016, Taipei, Taiwan.

[6] Specification of the Unbundled Smart Meter concept based on commercially existing smart meters (EN), project report of H2020 NOBELGRID, available at: www.nobelgrid.eu.

[7] Mihaela Albu, M. Sănduleac, Carmen Stanescu, "Syncretic use of smart meters for Power Quality monitoring in emerging networks", IEEE Transactions on Smart Grids, Volume 1, 2017

[8] Arbiter Systems, Inc., Arbiter1133a Data Sheet, available at www.arbiter.com/files/product-attachments/1133a.pdf

[9] Mihai Sănduleac, Carmen Stanescu, Nicolae Golovanov, "Power networks observability, control and automation using Unbundled Smart Meters", 2016 International Conference on Development and Application Systems (DAS), May 2016, Suceava.

[10] ENTSO-E Network Code / Commission regulation on establishing a guideline on electricity transmission system operation, voted in comitology on 4 May 2016 